\title{
Probable ancestral type of actinodont hinge in the Ordovician bivalve Pseudocyrtodonta Pfab, 1934
}

\author{
MARIKA STEINOVÁ
}

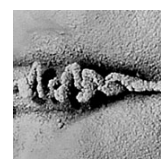

\begin{abstract}
The type species of Pseudocyrtodonta, P. ala and other two species P. incola, P. obtusa are known from the Middle and Upper Ordovician of the Prague Basin, Bohemia. Because of the actinodont type of the hinge, Pseudocyrtodonta is excluded from the subclass Protobranchia and is transferred to the Autobranchia, family Cycloconchidae. The family Pseudocyrtodontidae is considered invalid. Early and Middle Ordovician Cycloconchidae were highly diversified, containing 24 genera. Their diversity suddenly decreased during the Upper Ordovician to three genera only. The hinge of Pseudocyrtodonta could be considered morphologically close to the ancestral type of the hinge of the actinodonts. A complete species list of Ordovician actinodonts, including schematic figures of their hinge for the most important genera is presented. Pseudocyrtodonta was most probably an active burrower. - Key words: Bivalvia, Actinodontida, Cycloconchidae, Pseudocyrtodonta, Ordovician, Bohemia, systematics, palaeoecology.
\end{abstract}

SteINOVÁ, M. 2012. Probable ancestral type of actinodont hinge in the Ordovician bivalve Pseudocyrtodonta Pfab, 1934. Bulletin of Geosciences 87(2), 333-346 (5 figures, 1 table). Czech Geological Survey, Prague. ISSN 1214-1119. Manuscript received December 12, 2011; accepted in revised form January 26, 2012; published online March 23, 2012; issued March 30, 2012.

Marika Steinová, Czech Geological Survey, Klárov 3, P.O. Box 85, 11821 Praha 1, Czech Republic; marika.steinova@geology.cz

The Ordovician bivalves from Bohemia have been overlooked since Barrande (1881) and Pfab (1934) described them and their systematics should be revised. The preparation of the new Treatise on Invertebrate Paleontology for bivalves is in progress now and therefore it is an appropriate time for doing a revision of the Ordovician bivalves from Bohemia. Kř́̌ž \& Steinová (2009) began a revision with Hirnantian bivalves from Bohemia; this continues on well-preserved bivalves from the Middle Ordovician (early Darriwilian, Śárka Formation). First results reveal that the order Actinodontida Dechaseaux, 1952 in Bohemia is not confined only to the genus Redonia Rouault, 1851 but also to Pseudocyrtodonta Pfab, 1934.

Barrande (1881) figured Darriwilian Redonia bohemica, which clearly belongs to the Actinodontida. Barrande also figured two species, Leda ala and Leda incola, which Pfab (1934) considered as conspecific and assigned them to the new genus Pseudocyrtodonta. The other species that Pfab (1934) placed into Pseudocyrtodonta was P. obtusa (Barrande, 1881) from the Zahořany Formation (early Katian). Pfab (1934) defined Pseudocyrtodonta in his work about bivalves with taxodont hinge but he noticed that the hinge differs from the "normal" taxodont hinge. Maillieux (1939) described $P$. obtusa from Belgium and defined the new family Pseudocyrtodontidae. McAllester (1969) considered Pseudocyrtodonta as conspecific with Myoplusia Neumayr, 1884.
Bieler et al. (2010) and Carter et al. (2011) in their works, which are the basis for the new classification of bivalves, placed the Pseudocyrtodontidae into the Protobranchia Pelseneer, 1889 (Palaeotaxodonta Korobkov, 1954).

\section{Systematic palaeontology}

Abbreviations. $-\mathrm{V}=$ valve, $\mathrm{L}=$ length of the shell, $\mathrm{H}=$ height of the shell, $\mathrm{W}=$ width of the shell, $\mathrm{W} / 2$ = width of one valve (Kř́ž 1969). MBHR specimens deposited in the Museum of Dr. Bohuslav Horák, Rokycany; NM specimens deposited in the National Museum, Prague; LPB specimens deposited in the University of Western Brittany, Brest. PO specimens deposited in the collection of Marika Steinová in the Czech Geological Survey, Prague. All measurements are in millimetres.

Class Bivalvia Linné, 1758

Subclass Heterodonta Neumayr, 1884

Order Actinodontida Dechaseaux, 1952

Remarks. - In the Treatise on Invertebrate Paleontology (Newell 1969) Actinodontida was considered as synonym of Modiomorphoida Newell, 1969. Actinodontida differs 
from Modiomorphoida and they are important for early phylogeny of bivalves. There are three hypothesis about the early phylogeny of bivalves 1) some workers accept theory that actinodont teeth are derived from taxodont teeth (Babin 1966; Newell 1969; Pojeta 1971, 1978; Jell 1980; Babin \& Le Pennec 1982; Runnegar \& Bentley 1983; Pojeta \& Runnegar 1985; Waller 1990; Sánchez 1995, Cope 1996, 1997 and Ratter \& Cope 1998) 2) but some workers prefer an opposite view, namely that taxodont teeth are derived from actinodont teeth (Morris \& Fortey 1976, Morris 1980) 3) Sánchez \& Babin (1998) offered another alternative that palaeotaxodonts and palaeoheterodonts originated independently. Carter et al. (2000) confirm their hypothesis and showed that Actinodontida has apparently evolved convergently among Palaeotaxodonta. The oldest known species, which belongs to the Actinodontida is Intihuarella simplicidentata Sánchez in Sánchez \& Vaccari (2003) from Tremadocian of Argentina. Allen \& Sanders (1973) consider Prelametila Allen \& Sanders, 1973 as a possible living actinodont, but this has been dismissed by later authors (e.g. Cope 1997).

Actinodontida from the Ordovician (Table 1) were described from Argentina (Sánchez 1986, 1997, 2001, 2005; Sánchez \& Babin 1994, Sánchez \& Vaccari 2003, and Sánchez \& Benedetto 2007), Australia (Pojeta \& Gilbert Tomlinson 1977), China (Guo 1985, 1988; Fang \& Cope 2004, 2008), Bohemia (Barrande 1881, Pfab 1934), France (Rouault 1851, Barrois 1891, Babin 1966, Babin et al. 1982), North America (Meek 1871; Miller 1874; Foerste 1914; Ulrich 1893; Pojeta 1971, 1978), Spain (Babin \& Guttiérez-Marco 1985, 1991) and Wales (Salter 1859, Cope 1996).

Babin (1966) described some specimens from the Middle Ordovician of France, which he determined with uncertainty to Actinodonta Phillips, 1848. The type species of Actinodonta is A. cuneata Phillips, 1848 from the Silurian. Cope (2002) mentioned that this genus is problematic because Pojeta (1978) incorrectly recorded the type species as from the Ordovician. Furthermore, stratigraphical occurence of the genus Actinodonta is incorrectly recorded in the Treatise on Invertebrate Paleontology (Newell 1969) as the Middle Ordovician in spite of fact that, the type species Actinodonta cuneata occurs correctly in the Silurian. It seems that Actinodonta is confined to the Silurian and therefore Ordovician specimens from France need revision (Cope 2002). Babin (1966, fig. 59) figured schemes of the hinge in some species of Actinodontida. According to these schemes, hinge of "Actinodonta cuneata" from France differs from the type species, hinge of the "Actinodonta carinata" is very similar to Cycloconcha ovata but they differ in shape, and "Actinodonta obliqua" differs from other actinodonts in shape, which is more similar to lyrodesmatids.

Schemes of the Ordovician actinodont hinges are shown on the Fig. 1. The hinge of the family Pucamyidae is not figured, because the anterior part of the hinge is unknown.

Superfamily Anodontopsoidea Miller, 1889

Family Cycloconchidae Ulrich in Ulrich \& Scofield, 1894

Subfamily Cycloconchinae Ulrich in Ulrich \& Scofield, 1894

\section{Genus Pseudocyrtodonta Pfab, 1934}

Figures 2, 3, 4, 5

Type species. - P. ala (Barrande, 1881), Bohemia, Osek, Middle Ordovician, Darriwilian, Śárka Formation.

Diagnosis. - Equivalved, inequilateral, prosogyrate and posteriorly elongated cycloconchid. The dentition consists of numerous pseudocardinal teeth (looking like taxodont teeth) in anterior and one pseudolateral tooth in posterior part.

Description. - Shell equivalved, posteriorly elongated, inequilateral, with or without rostrate posterior. Umbos prosogyrate, projecting above the hinge margin, situated in the anterior half of the shell length. Anterior part of the shell is smaller than the posterior. Anterior margin is rounded, posterior margin elongated and also rounded, ventral margin convex.

Dentition (Fig. 2) with pseudocardinal teeth (pseudotaxodont) in anterior part, lying slightly below hinge line, one pseudolateral tooth in posterior part, long and slender, parallel to the hinge margin. Hinge continues below the umbos, without any gap. Anterior adductor muscle scar rounded or ovate, more pronounced than ovate posterior adductor muscle scar. Rounded pedal muscle scar in anterior part, joins with adductor muscle scar. Elongated pedal muscle scar in posterior part, separated from adductor muscle scar. Accessory muscle scars in the umbo region.

Discussion. - Pfab (1934) placed Pseudocyrtodonta in the Palaeotaxodonta, with a note that the hinge is different from "normal" taxodont hinge. Maillieux (1939) made Pseudocyrtodonta the type genus for the new family Pseudocyrtodontidae. McAlester (1969) included Pseudocyrtodonta in the synonymy of Myoplusia Neumayr, 1884. I was also of the opinion (Křiž \& Steinová 2009) that Pseudocyrtodonta obtusa belongs to Myoplusia, but in rediscovered type material of Barrande (1881) and also in the collection of J. Kř́̌ž (Czech Geological Survey) there are specimens from the Zahořany Formation (early Katian), which show actinodont hinges and the shape of the shell is very similar to Barrande's specimens of P. obtusa. Myoplusia and Pseudocyrtodonta from the Zahořany Formation are very similar in their general shape, but they differ in the type of the hinge, which is very often not well preserved. Myoplusia shows taxodont whereas Pseudocyrtodonta has an 

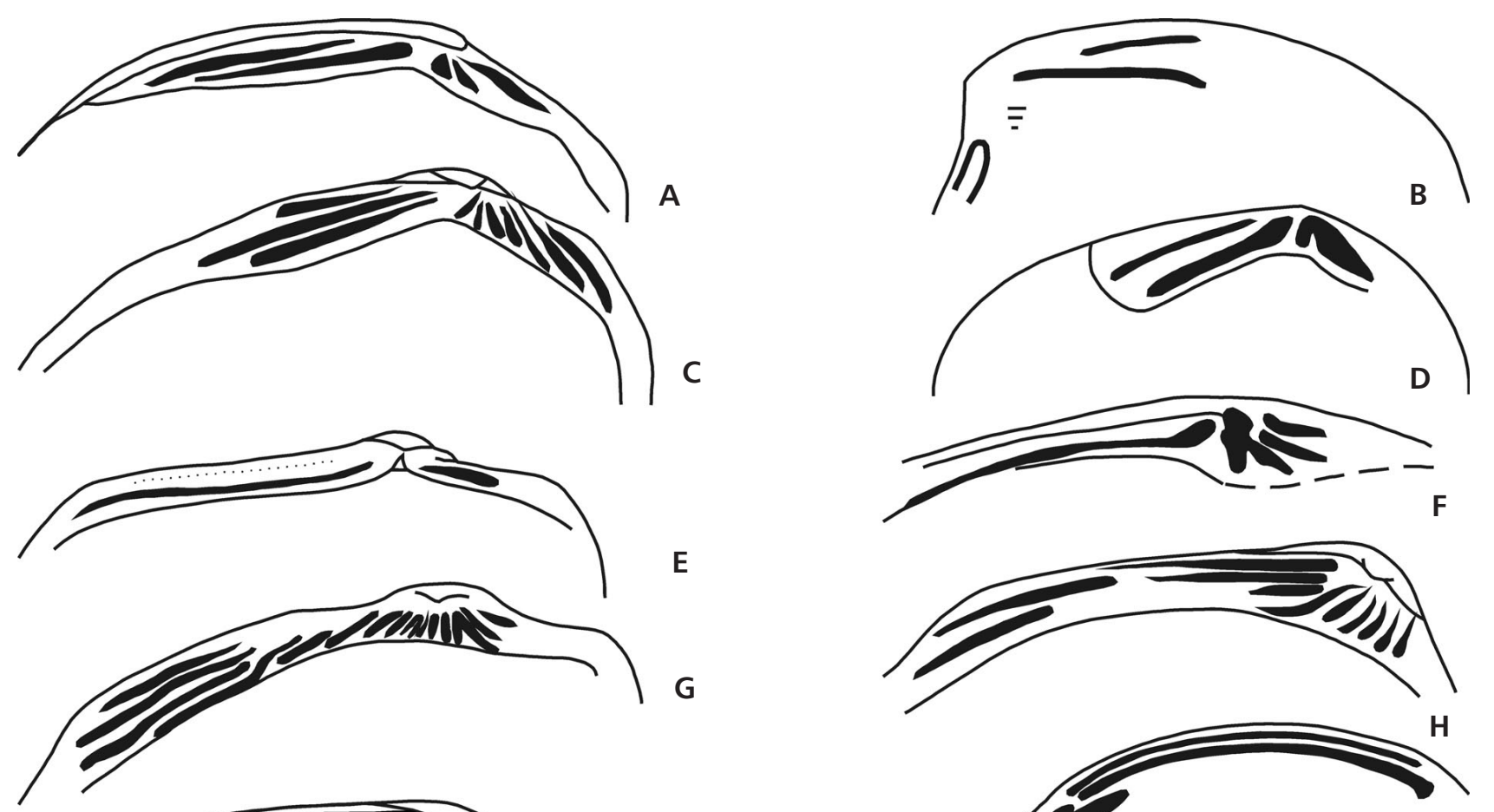

C
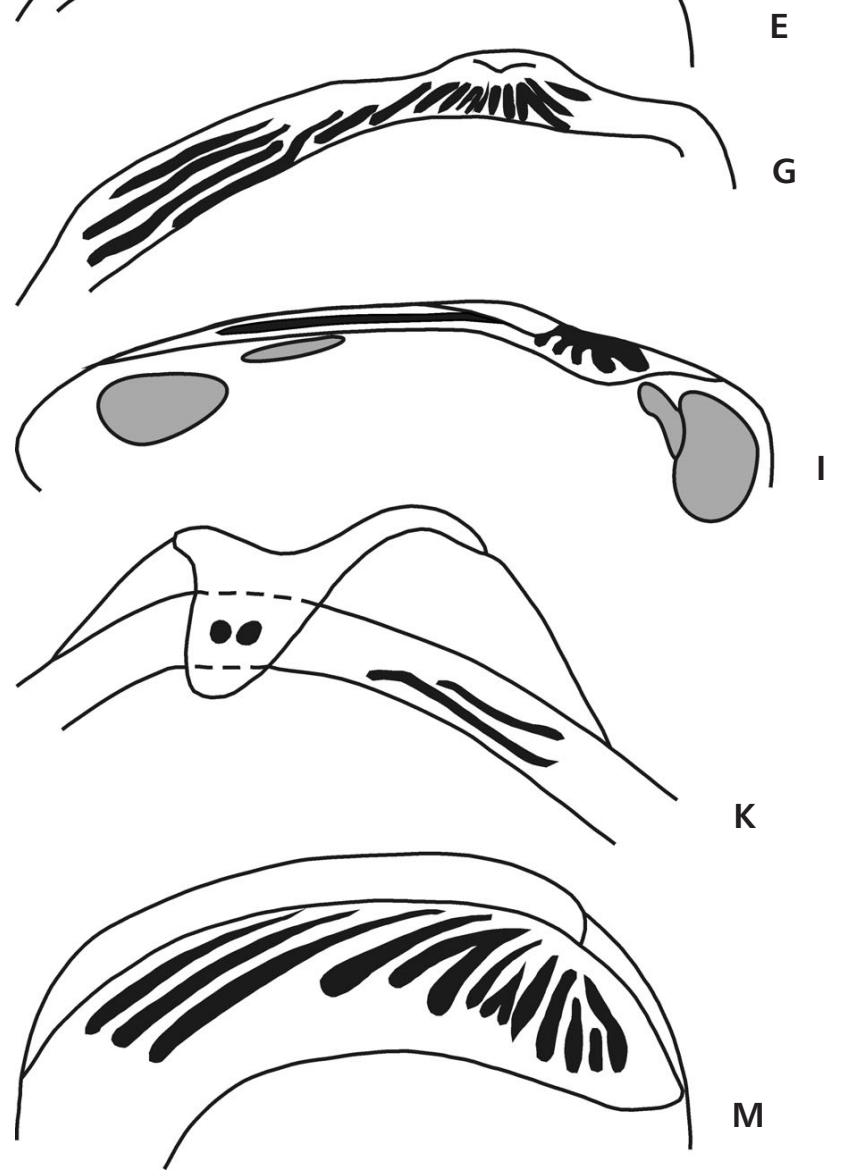

K

Figure 1. Hinge schemes of the important genera of the Ordovician Actinodontida. $-\mathrm{A}-\mathrm{I}$ - Cycloconchidae Ulrich, 1884; A - Cycloconcha, according to fig. 3 in Babin \& Gutiérrez-Marco (1985), left valve; B - Zhenxiongella, according to fig. 4 in Fang \& Cope (2008), right valve; C - Actinodonta, according to Treatise on Invertebrate Paleontology (1969), left valve; D - Famatinodonta, according to fig. 3 in Sánchez (2001), left valve; E - Fortowensia according to fig. 6D in Cope (1996), left valve; F - Poladonta, according to fig. 5 in Sánchez et al. (2003), left valve; G - Carminodonta, according to fig. 6A in Cope (1996), left valve; H - Ananterodonta, according to fig. 3C in Babin \& Gutiérrez-Marco (1985), left valve; I - Pseudocyrtodonta, according to specimen BHMR 14442, left valve. $\bullet$ J - Redoniidae Babin, 1966; Redonia according to specimen LPB 775 , right valve. $\bullet \mathrm{K}$ - Baidiostracidae Fang \& Cope, 2008; Baidiostraca, according to fig. 4 in Fang \& Cope (2008), right valve. $\bullet$ L, N - Intihuerallidae Sánchez, 2003; L - Cienagomya, according to fig. 3 in Sánchez (2005), left valve; N - Intihuarella, according to fig. 3 in Sánchez (2005), left valve. • M - Nyassidae Miller, 1877; Copidens according to fig. 3E in Babin \& Gutiérrez-Marco (1985), left valve. • O - Lametilidae Allen \& Sanders, 1973, Prelametila, according to fig. 36 in Allen \& Sanders, 1973, left valve. 


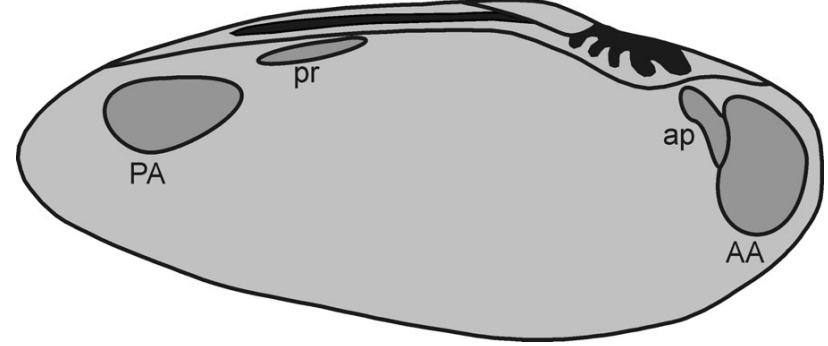

Figure 2. Pseudocyrtodonta incola, dorsolateral view showing hinge and muscle scars. Key to letter symbols: AA - anterior adductor muscle scar; PA - posterior adductor muscle scar; ap - anterior protractor muscle scar; $\mathrm{pr}$ - posterior retractor muscle scar.

actinodont type of hinge. So here the correction is made, Myoplusia obtusa described from the Kosov Formation (Kř̌̌ž \& Steinová 2009, pp. 421, 422, fig. 4F, G, I, J) should be classified as Myoplusia incisa (Barrande, 1881).

Bieler et al. (2010) and Carter et al. (2011) placed the Pseudocyrtodontidae in the Protobranchia Pelseneer, 1889 (Palaeotaxodonta). As mentioned above, the Pseudocyrtodontidae cannot be placed in the Palaeotoxodonta, because Pseudocyrtodonta has an actinodont hinge. Furthermore, there is no reason to keep family Pseudocyrtodontidae, which is not well defined. Its diagnosis from Mailieux (1939): "bivalves with dentition half taxodont and half heterodont" is common for the most part of the bivalves, which belong to Actinodontida. The genera with different types of the actinodont hinge are grouped in the family Cycloconchidae (Fig. 1A-I) and Pseudocyrtodonta does not show any unique characters for describing a new family. Therefore Pseudocyrtodonta is placed within the Cycloconchidae.

Relationships. - Ananterodonta Babin \& Guttiérrez-Marco, 1985 differs from Pseudocyrtodonta mainly in its hinge, which shows more numerous teeth in the posterior part and its anterior adductor muscle scar is more deeply impressed in the shell.

Mangbuina Guo, 1988 differs from Pseudocyrtodonta in the rounded shape of the shell, in the carina developed in the posterior part of the shell and in having three posterior teeth.

Zhenxiongella Fang \& Cope, 2008 differs from Pseudocyrtodonta in having two posterior teeth, small subumbonal teeth and only one anterior tooth. Anterior and posterior myophoric buttresses are characteristic.

Fasciculodonta Fang \& Cope, 2004 differs from Pseudocyrtodonta in its more deeply inserted adductor muscle scars and in its prominent shoulder in the posterior part of the shell.

Taselasmodum Guo, 1985 differs from Pseudocyrtodonta in having a more rounded shell, two elongated posterior teeth and more deeply impressed adductor scars.
Carminodonta Cope, 1996 differs from Pseudocyrtodonta in having a pronounced shoulder in the posterior part and in having more numerous teeth. Its hinge plate is wider than in Pseudocyrtodonta.

Fortowensia Cope, 1996 differs from Pseudocyrtodonta in having only one tooth in the anterior part.

Famatinodonta Sánchez, 2001 differs from Pseudocyrtodonta in having two strong pseudocardinals (one of them is in posterior part of the shell) and more pronounced adductor muscle scars.

Poladonta Sánchez in Sánchez et al. (2003) differs from Pseudocyrtodonta in having three pseudolateral teeth in the posterior part of the hinge.

Cycloconcha Miller, 1874 differs from Pseudocyrtodonta in having two pseudolateral teeth in posterior part and a more rounded shape.

Actinodonta Phillips, 1848 differs from Pseudocyrtodonta in having three pseudolateral teeth in the posterior part, more elongated anterior teeth, and more inserted anterior adductor muscle scars.

\section{Pseudocyrtodonta ala (Barrande, 1881)}

Figure 3

1881 Leda ala Barr.; Barrande, pl. 273, figs II/1-3.

1881 Synek antiquus Barr.; Barrande, pl. 275, figs I/1-2.

1968 Pseudocyrtodonta ala Barr. - McAlester, p. 48, pl. 29, figs 6-8.

Lectotype (SD, Pfab 1934). - Internal mould of the shell with conjoined valves, figured by Barrande (1881) on pl. 273 as figs II/1-3, NM L 27173. Other specimens on pl. 273, fig. II do not belong to Pseudocyrtodonta ala.

Type locality. - Bohemia, Prague Basin, Osek near Rokycany.

Type horizon. - Middle Ordovician, early Darriwilian, Sárka Formation.

Material. - 18 shells with conjoined valves, 10 right valves, and 8 left valves.

Diagnosis. - Pseudocyrtodonta with longitudinally elongated shell without prominent rostrum, and with less pronounced umbos.

Description. - Shell small (length maximally $9.6 \mathrm{~mm}$, height maximally $5.6 \mathrm{~mm}$ and width maximally $4 \mathrm{~mm}$ ), equivalve, inequilateral and longitudinally elongated, without prominent rostrum. Anterior part of the shell is smaller than the posterior part. Anterior margin is rounded, posterior margin elongated and also rounded, ventral 
Table 1. List of the Ordovician and early Silurian species belonging to order Actinodontida.

\begin{tabular}{|c|c|c|c|}
\hline Country & Species & Family & Stratigraphy \\
\hline \multirow{5}{*}{$\begin{array}{l}\text { Argentina (North Western } \\
\text { Argentina Basin) }\end{array}$} & Cienagomya bidentata Sánchez, 2005 & Intihuerallidae & Tremadocian, Floian \\
\hline & $\begin{array}{l}\text { Intihuarella simplicidentata } \\
\text { Sánchez \& Vaccari, } 2003\end{array}$ & Intihuerallidae & Tremadocian (Floresta Formation) \\
\hline & $\begin{array}{l}\text { Redonia condorensis Sánchez \& Benedetto, } \\
2007\end{array}$ & Redoniidae & Darriwilian \\
\hline & $\begin{array}{l}\text { Pseudoredonia radialis Sánchez \& Benedetto, } \\
2007\end{array}$ & Redoniidae? & Darriwilian \\
\hline & Pucamya wira Sánchez \& Benedetto, 2007 & Pucamyidae & Darriwilian \\
\hline Argentina (Sierra de Famatina) & Famatinodonta gonzaloi Sánchez, 2001 & Cycloconchidae & Floian/Dapingian (middle Arenigian) \\
\hline \multirow[t]{3}{*}{ Argentina (Western Argentina) } & Redonia riojana Sánchez, 1997 & Redoniidae & Floian/Dapingian (middle Arenigian) \\
\hline & Poladonta sanjuanina Sánchez et al., 2003 & Cycloconchidae & Sandbian \\
\hline & Redonia suriensis Sánchez \& Babin, 1994 & Redoniidae & Lower Ordovician \\
\hline Argentina (Provincia de Salta) & Cycloconcha cf. oblonga Foerste, 1914 & Cycloconchidae & Middle Ordovician \\
\hline Australia & $\begin{array}{l}\text { Copidens browni Pojeta \& Gilbert-Tomlinson, } \\
1977\end{array}$ & Nyassidae & Darriwilian (Nora Formation) \\
\hline \multirow[t]{4}{*}{ Bohemia } & Pseudocyrtodonta incola (Barrande, 1881) & Cycloconchidae & Darriwilian \\
\hline & Pseudocyrtodonta ala (Barrande, 1881) & Cycloconchidae & Darriwilian \\
\hline & Pseudocyrtodonta obtusa (Barrande, 1881) & Cycloconchidae & lower/middle Katian \\
\hline & Redonia deshayesi Rouault, 1851 & Redoniidae & Darriwilian \\
\hline \multirow[t]{4}{*}{ China (East Yunnan) } & Mangbuina prima Guo, 1988 & Cycloconchidae & Floian (Hongshiay Formation) \\
\hline & Zhenxiongella septata Guo, 1988 & Cycloconchidae & Floian (Hongshiay Formation) \\
\hline & Zadimerodia fastigiata Guo, 1988 & Nyassidae & Floian (Hongshiay Formation) \\
\hline & Baidiostraca aberrans Guo, 1988 & Baidiostracidae & Floian (Hongshiay Formation) \\
\hline \multirow[t]{4}{*}{ China (West Yunnan) } & Fasciculodonta impressa Fang \& Cope, 2004 & Cycloconchidae & Dapingian-Darriwilian (Upper Arenigian) \\
\hline & Fasciculodonta fengyiensis (Guo, 1985) & Cycloconchidae & Dapingian-Darriwilian (Upper Arenigian) \\
\hline & Taselasmodum decussatum Guo, 1985 & Cycloconchidae & Dapingian-Darriwilian (Upper Arenigian) \\
\hline & Yunnanoredonia laevis Fang \& Cope, 2004 & Redoniidae & Dapingian-Darriwilian (Upper Arenigian) \\
\hline \multirow[t]{8}{*}{ France (Armorican massive) } & Redonia deshayesi Rouault, 1851 & Redoniidae & Darriwilian (Shiste à Calymenes) \\
\hline & “Actinodonta cuneata” Phillips, 1848 & Cycloconchidae & Floian/Dapingian (Grès armoricain) \\
\hline & “Actinodonta carinata” Barrois, 1891 & Cycloconchidae & Floian/Dapingian (Grès armoricain) \\
\hline & “Actinodonta obliqua” Barrois, 1891 & Cycloconchidae & Floian/Dapingian (Grès armoricain) \\
\hline & “Actinodonta secunda” Barrois, 1891 & Cycloconchidae & Floian/Dapingian (Grès armoricain) \\
\hline & "Actinodonta acuta” Barrois, 1891 & Cycloconchidae & Floian/Dapingian (Grès armoricain) \\
\hline & “Actinodonta lata” Barrois, 1891 & Cycloconchidae & Floian/Dapingian (Grès armoricain) \\
\hline & “Actinodonta obtusa” Barrois, 1891 & Cycloconchidae & Floian/Dapingian (Grès armoricain) \\
\hline France (Montagne Noire) & Redonia michelae Babin et al., 1982 & Redoniidae & Floian and Dapingian \\
\hline \multirow[t]{4}{*}{ North America } & Cycloconcha ovata Ulrich, 1893 & Cycloconchidae & Upper Ordovician \\
\hline & Cycloconcha milleri (Meek, 1871) & Cycloconchidae & Upper Ordovician \\
\hline & Cycloconcha mediocardinalis Miller, 1874 & Cycloconchidae & Upper Ordovician \\
\hline & Cycloconcha oblonga Foerste, 1914 & Cycloconchidae & Upper Ordovician \\
\hline \multirow[t]{3}{*}{ Spain } & $\begin{array}{l}\text { Ananterodonta oretanica Babin \& } \\
\text { Gutiérrez-Marco, } 1985\end{array}$ & Cycloconchidae & Darriwilian (Schiste à Neseuretus) \\
\hline & Redonia deshayesi Rouault, 1851 & Redoniidae & Darriwilian \\
\hline & $\begin{array}{l}\text { Dulcineaia manchegana Babin \& } \\
\text { Gutiérrez-Marco, } 1991\end{array}$ & Redoniidae & Darriwilian \\
\hline \multirow[t]{6}{*}{ Wales (Llangyong Inlier) } & Actinodonta cuneata Phillips, 1848 & Cycloconchidae & Silurian (Llandovery) \\
\hline & Carminodonta crossi Cope, 1996 & Cycloconchidae & Floian \\
\hline & Fortowensia grandis Cope, 1996 & Cycloconchidae & Floian \\
\hline & Celtoconcha foveata Cope, 1996 & Cycloconchidae & Floian \\
\hline & Moridunia simplicidens Cope, 1996 & Redoniidae & Floian \\
\hline & Redonia anglica Salter, 1859 & Redoniidae & Floian/Dapingian (Arenigian) \\
\hline
\end{tabular}


margin convex. Less pronounced, strongly prosogyrate umbos are in the anterior half of the shell length. Hinge plate narrow with actinodont hinge, 2-4 pseudocardinal (pseudotaxodont) teeth in the anterior part (Fig. 3E, H), lying slightly below the hinge line. Single straight and slender pseudolateral tooth in the posterior part of hinge (Fig. 3E, H, L), and parallel to the hinge margin. Inner surface sculpture is smooth. Adductor muscle scars very badly preserved. Pedal and accessory muscle scars unknown. Outer surface sculpture and shell thickness is unknown.

Dimensions. -
Specimens
MBHR 2384a
MBHR 8902
MBHR 13159
MBHR 21039
MBHR 20368
MBHR 12701
MBHR 6334
MBHR 20567
MBHR 2420b
MBHR 14763
MBHR 4645
MBHR 18833
MBHR 20400
MBHR 20369
MBHR 21175
MBHR 18802
MBHR 7580
MBHR 20378
MBHR 20376
MBHR 18803
MBHR 20377
NM L 27173
MBHR 14293
MBHR 5067
MBHR 12908
MBHR 12826
MBHR 12647
MBHR 14294
MBHR 24036
MBHR 14236
MBHR 14421
MBHR 20410
MBHR 18840
MBHR 9774
MBHR 20411
MBHR 20345

Dimensions. -

Specimens

MBHR 8902

MBHR 13159

BHR 2103

MBHR 6334

MBHR 4645

BHR 18833

MBHR 21175

MBHR 18802

MBHR 20378

MBHR 20376

MBHR 20377

NM L 27173

HR 14293

MBHR 12908

MBHR 12826

BHR 12647

MBHR 24036

MBHR 14236

MBHR 18840

MBHR 20345

$\begin{array}{cccc}\text { V } & \text { L } & \text { H } & \text { W/2 } \\ \text { A } & 9.6 & 5.6 & 2.0 \\ \text { A } & 9.6 & 5.1 & 1.4 \\ \text { R } & 7.9 & 4.9 & - \\ \text { A } & 7.8 & 4.9 & 1.5 \\ \text { A } & 7.8 & 4.8 & 1.5 \\ \text { R } & 7.6 & 4.1 & - \\ \text { R } & 7.5 & 4.5 & - \\ \text { L } & 7.3 & 3.0 & - \\ \text { L } & 7.5 & 5.0 & 1.4 \\ \text { R } & 7.1 & 5.0 & - \\ \text { L } & 7.1 & 4.9 & 1.4 \\ \text { L } & 6.9 & 4.8 & - \\ \text { A } & 6.9 & 4.5 & 1.3 \\ \text { A } & 6.8 & 4.5 & 1.5 \\ \text { A } & 6.6 & 3.9 & 1.2 \\ \text { L } & 6.1 & 5.0 & - \\ \text { A } & - & 4.0 & 1.0 \\ \text { A } & 6.1 & 4.0 & 1.0 \\ \text { A } & 6.0 & 4.0 & 1.1 \\ \text { L } & 6.0 & 3.9 & - \\ \text { A } & 5.9 & 3.9 & 1.0 \\ \text { A } & 5.9 & 3.8 & - \\ \text { L } & 5.9 & 2.8 & - \\ \text { R } & 5.9 & 4.0 & - \\ \text { A } & 5.8 & 3.5 & 1.0 \\ \text { A } & 5.6 & 3.2 & 0.9 \\ \text { R } & 5.2 & 3.5 & - \\ \text { A } & 5.1 & 3.5 & 1.0 \\ \text { L } & 5.0 & 3.5 & - \\ \text { R } & 5.0 & 2.9 & 1.3 \\ \text { R } & 4.5 & 2.5 & - \\ \text { A } & 4.2 & 3.0 & 1.2 \\ \text { R } & 4.1 & 2.9 & - \\ \text { R } & 3.8 & 2.1 & 0.2 \\ \text { A } & 3.1 & 2.1 & 0.4 \\ \text { A } & 2.8 & 1.4 & 0.4 \\ & & & \end{array}$

Discussion. - Barrande (1881) figured two species from the Šárka Formation Leda ala and Leda incola. Pfab (1934) regarded them as conspecific. Here the view is taken, following the opinion of Barrande (1881), that they are different. They differ in shape, $P$. incola is more longitudinally elongated and has a rostrate posterior part. The umbos in $P$. incola are more pronounced than in $P$. ala. The hinge seems to be very similar, with one pseudolateral tooth in posterior part and several small pseudocardinal (pseudotaxodont) teeth in anterior part. However, the hinge in $P$. ala is ill-preserved. McAlester (1968) figured as the lectotype of Pseudocyrtodonta ala (pl. 29, figs 6-8), the specimen from the collection of National Museum, Prague but it differs from Barrande's figures in having a damaged hinge, contrary to the specimen figured by Barrande (1881) and designated by Pfab (1934) as the lectotype. Fasciculodonta impressa Fang \& Cope, 2004 from West Yunnan of China (Dapingian/Darriwilian) seems to be similar to $P$. ala in its longitudinally elongated shell, prosogyrate umbos and mainly in its hinge with one pseudolateral tooth in the posterior part and numerus pseudocardinal teeth in the anterior part. But $F$. impressa differs from $P$. ala in having more deeply impressed adductor muscle scars and in having a prominent shoulder in the posterior part of the shell.

Occurrence. - Bohemia, Prague Basin, early Darriwilian: Díly, Díly 1, Osek near Rokycany, Osek 1, Rokycany, Rokycany 2 - near cemetery, Rokycany 17.

\section{Pseudocyrtodonta incola (Barrande, 1881) Figure 4}

1881 Leda incola Barr.; Barrande, pl. 270, figs III/1-4. 1934 Pseudocyrtodonta ala Barr. - Pfab, p. 237, pl. III (IV), fig. 13.

Lectotype (SD, herein). - Internal mould of the shell with conjoined valves, figured by Barrande (1881) on pl. 270, as figs III/1-4, NM L 27117. Other specimens figured on the pl. 270, fig. III do not belong to $P$. incola.

Type locality. - Bohemia, Prague Basin, Osek near Rokycany.

Figure 3. A-Q - Pseudocyrtodonta ala (Barrande, 1881). A, B - articulated specimen, NM L27173, lectotype; A - dorso-lateral view, $\times 9.7$; B - left lateral view, $\times 6.4$. $\bullet \mathrm{C}, \mathrm{L}$ - right valve, MBHR 6334; C - lateral view, $\times 4.1$; $\mathrm{L}$ - dorsolateral view, pseudolateral tooth in the posterior part, $\times 8.1$. $\bullet$ - articulated specimen, MBHR 21039, left lateral view, $\times 9.1 \cdot \bullet \mathrm{E}-\mathrm{G}-$ articulated specimen, MBHR 2384a; E-dorsal view, pseudocardinal teeth in anterior part and pseudolateral tooth in posterior part, $\times 18.2 ; \mathrm{F}$ - right lateral view, $\times 5.9 ; \mathrm{G}-$ left lateral view, $\times 4.6 . \bullet \mathrm{H}, \mathrm{I}-$ right valve, MBHR $12701 ; \mathrm{H}-$ dorsal view, long tooth in posterior part, two pseudocardinals in anterior part, $\times 4.6$; I - right lateral view, $\times 8.2$. $\bullet \mathrm{J}-$ left valve, MBHR 18803 , left lateral view with part of the pseudolateral tooth in the posterior, $\times 6.4 \cdot \bullet \mathrm{K}, \mathrm{P}-$ articulated specimen, MBHR 12826; $\mathrm{K}-$ dorsal view, $\times 10.5 ; \mathrm{P}-$ right lateral view, $\times 7.2 . \bullet \mathrm{M}, \mathrm{O}-$ articulated specimen, MBHR 20373; M - left lateral view, $\times 4.3$; O - right lateral view, $\times 6.6 . \bullet \mathrm{N}, \mathrm{Q}-$ articulated specimen, MBHR 8902; N - dorsal view, $\times 5$; Q - right lateral view, $\times 6$. • A-B, D - Prague Basin, Bohemia, Osek locality, early Darriwilian. • C, L - Prague Basin, Bohemia, Díly locality, early Darriwilian. • E-G, N, Q - Prague Basin, Bohemia, Rokycany locality, early Darriwilian. • H, I - Prague Basin, Bohemia, Osek 1 locality, early Darriwilian. - J - Prague Basin, Bohemia, Díly 1 locality, early Darriwilian. • K, P - Prague Basin, Bohemia, Díly 3 locality, early Darriwilian. 
Marika Steinová • Probable ancestral type of actinodont hinge in the Ordovician bivalve Pseudocyrtodonta

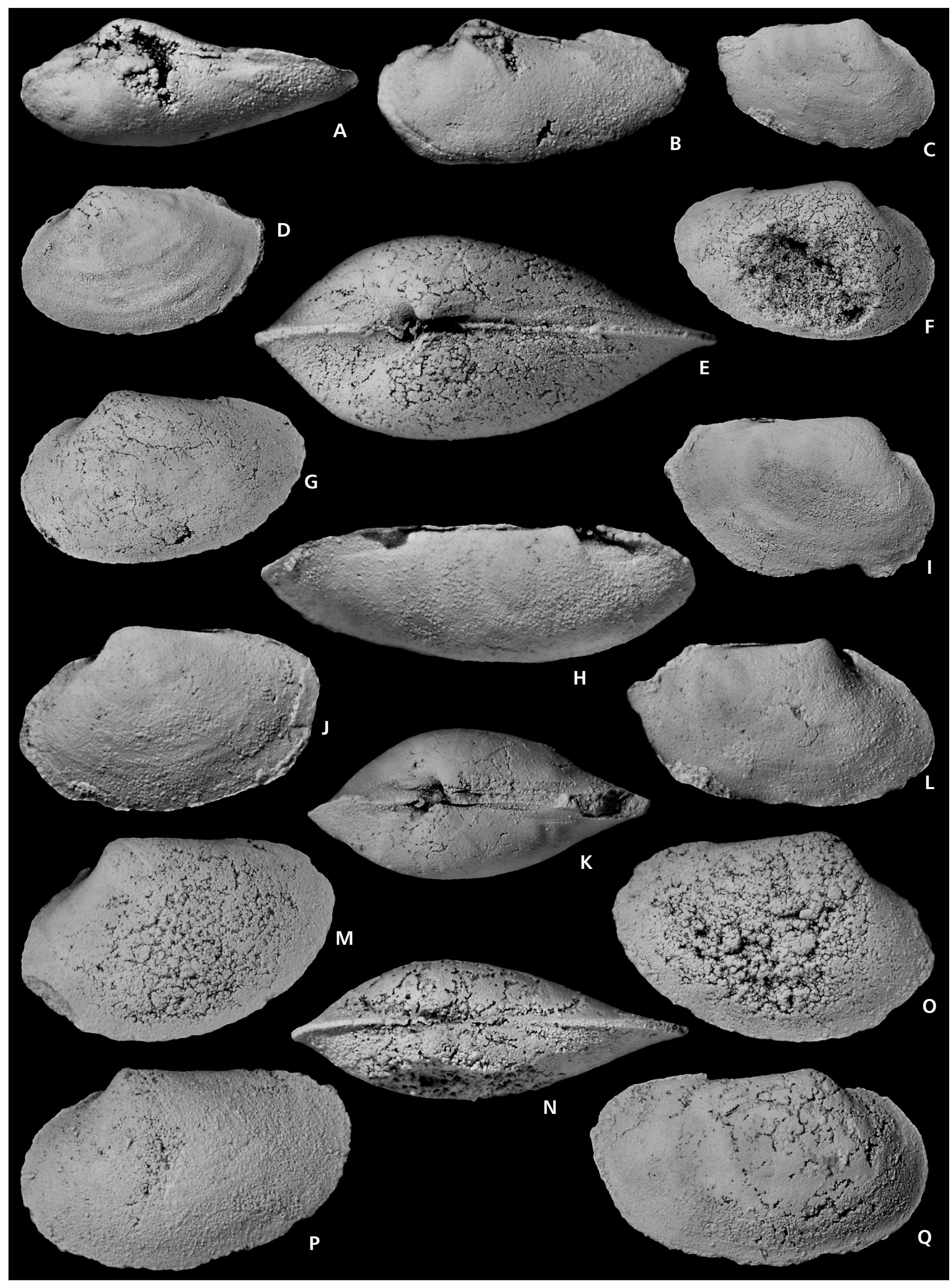


Type horizon. - Middle Ordovician, early Darriwilian, Šárka Formation.

Material. - 44 shells with conjoined valves, 20 right valves, and 11 left valves.

Diagnosis. - Pseudocyrtodonta with longitudinally elongated shell, with rostrate posterior part and with more pronounced umbos.

Description. - Shell small (length maximally $9 \mathrm{~mm}$, height maximally $5 \mathrm{~mm}$ and width maximally $3.2 \mathrm{~mm}$ ), equivalve, inequilateral and longitudinally elongated, with rostrate posterior part. More pronounced, strongly prosogyrate umbos situated in the anterior part of the shell. Anterior margin of the shell rounded, posterior margin rostrate, ventral margin convex. Hinge actinodont, in the anterior part 3-6 pseudocardinal (pseudotaxodont) teeth (Fig. 4C, H, K), lying under hinge line. After Babin (1966) anterior teeth can be classified as convexodont. In the posterior part of the shell one straight, slender pseudolateral tooth (Fig. 4G). Anterior adductor muscle scar is almost elliptic, more deeply impressed than elliptic posterior adductor muscle scar. Rounded pedal muscle scar in anterior part, joins with adductor muscle scar (Fig. 4K, P). Elongated pedal muscle scar in posterior part, separated from adductor muscle scar (Fig. 4K). In the umbo region of the lectotype two accessory narrow elliptic muscle scars (Fig. 4A) are developed. Inner surface sculpture is smooth. Shell thickness and outer surface sculpture unknown.

Dimensions. -

Specimens

MBHR 13340

MBHR 20341

NM L 40913

MBHR 21193

MBHR 4166

NM L 40914

MBHR 14763

MBHR 12569

NM L 27117

MBHR 20393

$\begin{array}{lr}\text { V } & \text { L } \\ \text { A } & 9.0 \\ \text { A } & 8.1 \\ \text { L } & 8.0 \\ \text { R } & 7.8 \\ \text { A } & 7.5 \\ \text { L } & 7.1 \\ \text { R } & 7.1 \\ \text { A } & 7.0 \\ \text { A } & 6.9 \\ \text { A } & 6.8\end{array}$

MBHR 13527

MBHR 4727a

MBHR 4726

MBHR 12423

MBHR 20354

MBHR 12723

MBHR 20353

MBHR 20459

MBHR 13452

MBHR 55902

NM L 40915

MBHR 20350

MBHR 2384b

MBHR 20370

MBHR 8980

MBHR 12432

MBHR 13140

MBHR 20355

NM L 40916

MBHR 20344

MBHR 20356

MBHR 13415

MBHR 13538

NM L 40917

MBHR 14840

MBHR 20357

MBHR 20460

MBHR 5820

MBHR 19516

MBHR 17795

MBHR 20358

MBHR 20374

NM L 40918

MBHR 14696

MBHR 14580

MBHR 55918

MBHR 20395

MBHR 14442

MBHR 20375

MBHR 14299

MBHR 14352a

MBHR 19207

MBHR 20373

MBHR 18813

MBHR 20398

MBHR 20494

MBHR 14273

MBHR 20359

MBHR 13368
$\mathrm{R}$

$\mathrm{R}$

$\mathrm{R}$

A

A

A

A

A

$\mathrm{R}$

A

$\mathrm{R}$

A

A

A

R

A

L

A

A

A

A

R

A

A

$\mathrm{R}$

A

A

$\mathrm{R}$

A

L

$\begin{array}{lll}6.5 & 4.5 & - \\ 6.5 & 3.9 & 1.0 \\ 6.5 & - & - \\ 6.2 & 2.9 & 1.6 \\ 6.2 & 3.1 & 1.0 \\ 6.1 & 3.0 & 0.9 \\ 6.0 & 3.5 & 1.0 \\ 6.0 & 3.1 & 0.9 \\ 6.0 & 3.0 & - \\ 6.0 & 3.0 & 0.6 \\ 5.9 & 3.4 & - \\ 5.9 & 3.1 & 1.0 \\ 5.9 & 3.0 & 0.8 \\ 5.9 & 2.9 & 0.9 \\ 5.9 & 2.7 & - \\ 5.8 & 3.1 & 1.0 \\ 5.8 & 3.0 & - \\ 5.8 & 3.0 & 0.9 \\ 5.7 & 3.4 & 0.8 \\ 5.7 & 3.1 & 0.8 \\ 5.6 & 2.8 & 0.9 \\ 5.5 & 3.5 & 1.2 \\ 5.5 & 3.1 & 0.9 \\ 5.5 & 2.9 & 0.9 \\ 5.5 & 3.1 & - \\ 5.5 & 2.9 & 1.0 \\ 5.5 & 2.9 & 0.6 \\ 5.5 & 2.8 & - \\ 5.4 & 2.9 & 0.7 \\ 5.4 & 2.5 & - \\ 5.3 & 3.2 & 1.0 \\ 5.2 & 3.0 & 0.9 \\ 5.2 & 2.9 & 1.0 \\ 5.2 & 2.9 & - \\ 5.1 & 3.5 & - \\ 5.1 & 3.1 & - \\ 5.1 & 2.9 & 0.7 \\ 5.1 & 2.9 & - \\ 5.1 & 2.8 & 0.7 \\ 5.1 & 2.1 & - \\ 5.0 & 3.0 & - \\ 5.0 & 2.9 & - \\ 5.0 & 2.9 & 0.7 \\ 5.0 & 2.9 & 0.5 \\ 5.0 & 2.6 & 0.7 \\ 5.0 & 2.6 & 0.7 \\ 5.0 & 2.5 & - \\ 4.9 & 2.9 & 0.8 \\ 4.8 & 3.0 & 0.7\end{array}$

Figure 4. A-Q-Pseudocyrtodonta incola (Barrande, 1881). A-C - articulated specimen, NM L27117, lectotype; A - left lateral view with two accessory muscle scars in the umbo region, $\times 5.4$; B - right lateral view, $\times 5.2$; C - dorsal view with actinodont hinge, adductor muscle scars with pedal muscle scars, $\times 10.3$. $\bullet$ D , G - right valve, MBHR $13415 ; \mathrm{D}$ - detail of the pseudolateral tooth in posterior part, $\times 8.2 ; \mathrm{G}-$ right lateral view with lateral tooth in the posterior part, $\times 6.3 . \bullet \mathrm{E}, \mathrm{F}, \mathrm{M}-$ articulated specimen, MBHR 20355; E - right lateral view with pseudolateral tooth in posterior and pseudocardinal teeth in anterior, $\times 3.4 ; \mathrm{F}$ - dorsal view with actinodont hinge, $\times 9.4 ; \mathrm{M}-$ left lateral view, $\times 3.5 . \bullet \mathrm{H}, \mathrm{I}-$ articulated specimen, $\mathrm{MBHR} 12432$; $\mathrm{H}-$ dorsal view with actinodont hinge, $\times 10.3$; I - right lateral view with anterior adductor muscle scar, $\times 5.2 . \bullet J, L, N-$ articulated specimen, MBHR $2384 \mathrm{~b} ; \mathrm{J}-$ left lateral view, $\times 7.1 ; \mathrm{L}-$ right lateral view, $\times 7.3 ; \mathrm{N}$ - dorsal view with actinodont hinge, $\times 7.6 . \bullet \mathrm{K}-$ left valve, MBHR 14442 , dorsolateral view with pseudolateral tooth in posterior and pseudocardinal teeth in anterior, anterior adductor muscle scar with pedal muscle scar. $\times 7.2$. $\bullet$ O-Q - articulated specimen, MBHR 20353; O - left lateral view, $\times 7.2 ; \mathrm{P}$ - dorsal view with actinodont hinge, adductor muscle scars with pedal muscle scars, $\times 11.4$; $\mathrm{Q}-$ right lateral view, $\times 6.6$. A-C - Prague Basin, Bohemia, Osek locality, early Darriwilian. $\bullet$ D, G - Prague Basin, Bohemia, Díly 2 locality, early Darriwilian. - E, F, K, M, O-Q - Prague Basin, Bohemia, Díly 1 locality, early Darriwilian. • H, I - Prague Basin, Bohemia, Osek 1 locality, early Darriwilian. - J, L, N - Prague Basin, Bohemia, Rokycany locality, early Darriwilian. 
Marika Steinová • Probable ancestral type of actinodont hinge in the Ordovician bivalve Pseudocyrtodonta

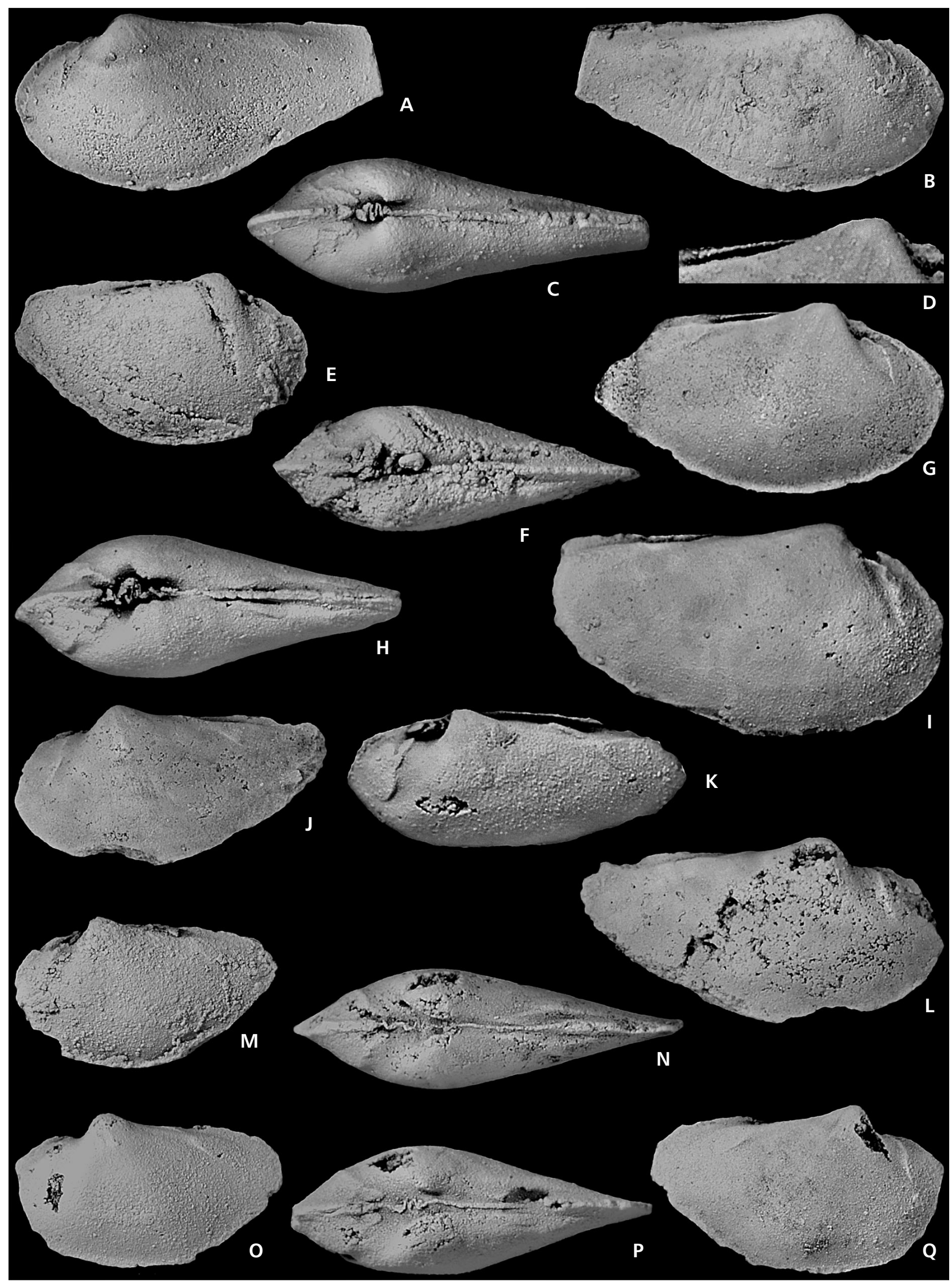




MBHR 20399
MBHR 4727b
MBHR 55096
MBHR 20461
MBHR 14838
MBHR 14295
MBHR 14450
MBHR 14429a
MBHR 14429b
MBHR 14297
MBHR 14352b
MBHR 20462
MBHR 20371
MBHR 19129
MBHR 14764

$\begin{array}{ll}\text { A } & 4.5 \\ \text { A } & 4.5 \\ \text { L } & 4.3 \\ \text { A } & 4.2 \\ \text { L } & 4.0 \\ \text { L } & 4.0 \\ \text { R } & 4.0 \\ \text { L } & 3.9 \\ \text { R } & 3.9 \\ \text { R } & 3.8 \\ \text { R } & 3.8 \\ \text { A } & 3.5 \\ \text { A } & 3.0 \\ \text { A } & 2.0 \\ \text { R } & 1.8\end{array}$

Occurrence. - Bohemia, Prague Basin, early Darriwilian: Díly, Díly 1, Díly 2, Díly 3, Díly 4 - south slope of Hůrka, Díly 6, Drahouš 3, Drahouš 4, Mýto 1, Osek near Rokycany, Osek 1, Pětidomky, Rokycany, Rokycany 2, Rokycany 17, Šárka cihelna, Těškov 1.

\section{Pseudocyrtodonta obtusa (Barrande, 1881)} Figure 5

1881 Nucula obtusa Barr.; Barrande, pl. 272, figs I/1-17. 1934 Pseudocyrtodonta obtusa Barr. - Pfab, p. 238, pl. III (IV), fig. 12.

Lectotype (SD, herein). - Internal mould of the shell with conjoined valves, figured by Barrande (1881) on pl. 272, as figs I/12-14, NM L22683.

Paralectotypes. - Other four internal moulds of shells with conjoined valves, figured by Barrande (1881) on pl. 272, as figs I/1-4, 5-8, 9-11, 15-17, NM L 27150, NM L 22684, NM L 22685, NM L 22686.

Type locality. - Bohemia, Prague Basin, Štěrboholy.

Type horizon. - Upper Ordovician, early Katian, Zahořany Formation.

Material. - 4 shells with conjoined valves, 1 right valve, and 2 left valves.
Diagnosis. - Pseudocyrtodonta with obese shells having pronounced projection in anterior part, large and strong pseudotaxodont teeth in anterior part increasing anteriorly.

Description. - Shell small (length maximally $11.1 \mathrm{~mm}$, height maximally $7.8 \mathrm{~mm}$ and width maximally $5.8 \mathrm{~mm}$ ), equivalve, inequilateral, obese and slightly longitudinally elongated. Anterior and posterior margin of the shell rounded. Anterior part is much smaller than posterior part. A strong projection is developed in the anterior part. Strongly prosogyrate umbos are situated in the anterior part of the shell. Hinge actinodont, in the anterior part with 3-5 pseudocardinal (pseudotaxodont) teeth, lying under hinge line. The most anterior teeth are larger (Fig. 5D, J, M). In the posterior part of the shell one straight pseudolateral tooth (Fig. 5J, M) is developed parallel to the hinge margin. Anterior adductor muscle scar rounded (Fig. 5F, I). Posterior adductor muscle scar not well preserved. In the umbo region of lectotype three accessory rounded muscle scars (Fig. 5J) are developed. Inner surface sculpture is smooth. Shell thickness and outer surface sculpture unknown.

Dimensions. -

Specimens

PO 1

$\mathrm{PO} 2$

NM L 22684

NM L 22686

NM L 22683

NM L 27150

NM L 22685

V
L
L
A
R
A
A
A

L
11.1
10.5
9.3
8.4
8.2
7.5
-

$\mathrm{H}$
7.8
6.8
6.4
6.6
6.0
5.5
6.5

$\mathrm{W} / 2$

Discussion. - Maillieux (1939) described and figured P.obtusa from the Middle Ordovician (Darriwilian). It is not possible to observe actinodont hinge on the figured specimen. Also in his description of P. obtusa it is mentioned that hinge in the Belgian specimens is not preserved. It is here considered that the shape of the shell is also completely different and therefore it is not possible to determine this specimen as P. obtusa. P. obtusa is similar to Myoplusia incisa (from the Zahořany Formation, early Katian), but they differ in hinge, which is taxodont in $M$. incisa. When the hinge is not preserved it is possible to distinguish them from the pronounced projection in the anterior part, which is developed in $P$. obtusa. P. obtusa differs from $P$. ala and P. incola in having

Figure 5. A-N - Pseudocyrtodonta obtusa (Barrande, 1881). • A, H, C-articulated specimen, NM L 27150, paralectotype; A - left lateral view, $\times 6.2$; $\mathrm{H}$ - right lateral view, $\times 7.7$; C - dorsal view, $\times 11 . \bullet \mathrm{B}, \mathrm{E}, \mathrm{F}, \mathrm{I}-$ articulated specimen, NM L 22684, paralectotype; B - left lateral view, $\times 8.4$; E - dorsal view with pseudocardinal teeth in anterior and pseudolateral tooth in posterior, $\times 12.1 ; \mathrm{F}-$ detail of the anterior adductor muscle scar, $\times 9.3$; I - right lateral viewwith anterior adductor muscle scar. $\bullet \mathrm{D}$ - articulated specimen, NM L22686, paralectotype, dorsal view with pseudocardinal teeth, the most anterior tooth is the largest, $\times 8.8 . \bullet \mathrm{G}, \mathrm{J}, \mathrm{K}-$ articulated specimen, NM L 22683 , lectotype; $\mathrm{G}-$ left lateral view, $\times 7.7$; - dorsal view with actinodont hinge $\times 16 ; \mathrm{K}$ - right lateral view with anterior adductor muscle scar, $\times 8.1$. $\bullet \mathrm{L}-\mathrm{N}$ - articulated specimen, NM L 22685, paralectotype; $\mathrm{L}-$ left lateral view with adductor muscle scar, $\times 5.2 ; \mathrm{M}$ - dorsal view with actinodont hinge, $\times 10.5 ; \mathrm{N}$-right lateral view, $\times 8.8$. $・ \mathrm{~A}, \mathrm{H}, \mathrm{C}-\mathrm{Prague}$ Basin, Bohemia, Loděnice locality, early Katian. • B, E-G, I-N - Prague Basin, Bohemia, Štěrboholy locality, early Katian. • D - Prague Basin, Bohemia, Butovice locality, early Katian. 
Marika Steinová • Probable ancestral type of actinodont hinge in the Ordovician bivalve Pseudocyrtodonta

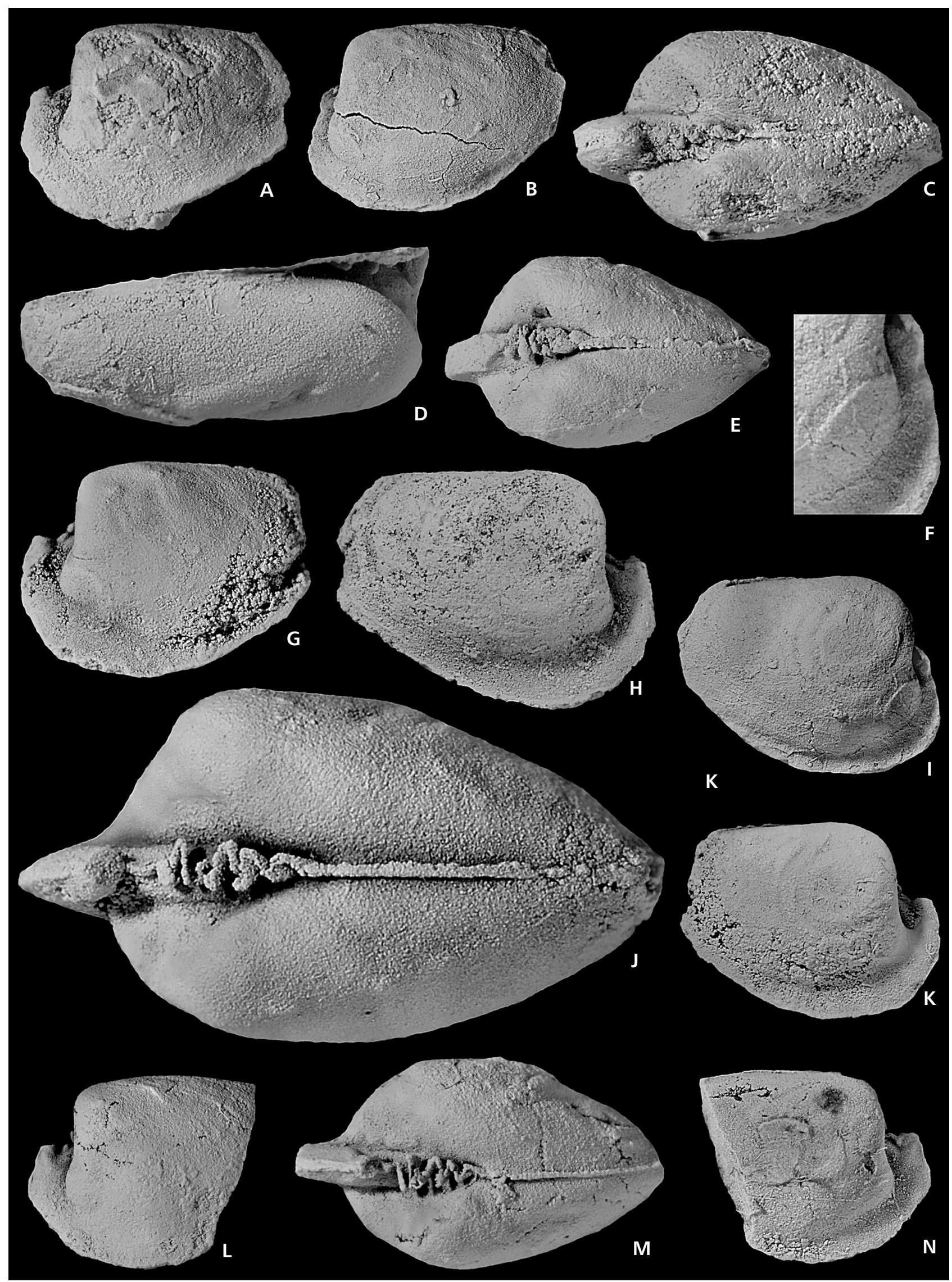


more obese and rounded shells, the anterior teeth are larger and stronger, the adductor muscle scars are rounded, and the shell has an anterior projection.

Occurrence. - Bohemia, Prague Basin, early Katian: Butovice, Loděnice, Štěrboholy.

\section{Palaeoecology}

Babin \& Guttiérrez-Marco (1991) considered actinodonts from Spain (Redonia and Ananterodonta) as shallow infaunal filter feeders (mentioning one specimen of Redonia with bryozoan incrustation supporting the idea that it was probably partly projected above the sediment-water interface). Allen \& Sanders (1973) described two interesting recent families Lametilidae and Siliculidae. They consider Lametilidae and mainly genus Prelametila closely related to the bivalves with actinodont hinge consider Prelametila as possible living actinodont. Cope (1997) showed that this pseudo-actinodont dentition developed by fusing of teeth of nuculoids in one case and development of teeth is solemyoids-like forms in another case. These forms are not related to the actinodonts, but their hinge are somewhat similar. After Allen \& Sanders (1973) taxodont teeth lock the valves together, distal elongate teeth are able to roll against one another. Therefore the tendency of the valves to shear is thus reduced. Because of the relatively equilateral shell, the more inflated form and small foot they consider Prelametila as not an active burrower. They are relatively sedentary forms, possibly lying in horizontal or near horizontal position close to the sediment surface. All the actinodonts known from the Ordovician have the umbo moved to the anterior part of the shell. Pseudocyrtodonta is not an exception. Pseudocyrtodonta is more similar to Silicula Jeffreys, 1879 in the shape of the shell. Silicula is considered to be active burrower. It has less inflated shell with the umbo in the anterior part, and large foot. It is also supposed that Pseudocyrtodonta was active infaunal burrower. This opinion is also supported by the common preservation of the shells with conjoined valves.

\section{Diversification}

Pseudocyrtodonta is the other genus newly determined as belonging to the Cycloconchidae and confirms that cycloconchoids were highly diversified during the Early and Middle Ordovician. Actinodonts in the Upper Ordovician are less diversified but it could be caused also by the lack of knowledge about bivalves from this series. Only Poladonta, Cycloconcha and Pseudocyrtodonta are known from the Upper Ordovician. Cope (2002) showed that heteroconchian bivalves are particularly characteristic of high latitudes and few Upper Ordovician high latitude faunas have been described.

\section{Phylogenetic considerations - probable ancestral type of actinodont hinge?}

This question is very important for the early evolution of bivalves - did the actinodont teeth evolve from the palaeotaxodont teeth and/or vice versa, or are these groups independent. The oldest known actinodont Intihuarella has got very simple hinge (Fig. 1N) and differs from other actinodonts in not having pseudocardinals. Therefore it is an uncertain if this genus is correctly determined to Actinodontida.

Pseudocardinal teeth are similar to taxodont teeth (sometimes they are called also pseudotaxodont teeth), mainly in some genera (Fasciculodonta and Pseudocyrtodonta) and could show close relationships between actinodonts and palaeotaxodonts. Fang \& Cope (2004) consider the hinge of Fasciculodonta (numerous pseudocardinals in anterior part and one pseudolateral in posterior part of the shell) as close to the ancestral type for the actinodonts, if the group was derived from the cardiolarioids. Pseudocyrtodonta from Bohemia possesses a very similar type of hinge to Fasciculodonta. Pseudocyrtodonta obtusa from the Upper Ordovician has almost the same hinge as $P$. ala and $P$. incola from the Middle Ordovician, and therefore it seems that the type of hinge is a very conservative feature. However, Carter et al. (2001) in their cladisitic analysis support the theory about independent groups, they still place Cardiolaria (Cardiolaridae Cope, 1997, Protobranchia) close to actinodonts. Also the lack of information about bivalves from the Furongian and Lower Ordovician leaves the question about early evolution of bivalves still open. Fang \& Cope (2008) discussed some phylogenetic considerations between cycloconchoids. Also they conclude that in general, determination of the relationships between taxa is hampered by the lack of material from many localities. Pseudocyrtodonta from Bohemia with the simple hinge and shell shape is closely related to Fasciculodonta from West Yunnan, China.

\section{Conclusions}

(i) Family Pseudocyrtodontidae Maillieux, 1939, which is assigned in the new Classification System for Bivalvia (Carter et al. 2011) as a member of the Protobranchia, is not correct. Pseudocyrtodonta is herein shown to belong to the Cycloconchidae and contains three species: $P$. ala, and $P$. incola from the early Darriwilian and P. obtusa from the early Katian.

(ii) In the Early and Middle Ordovician the family Cycloconchidae is highly diversified (24 genera), but is 
much less diversified in the Upper Ordovician (3 genera). The decrease in diversity could be artificial and may be caused by the lack of the information about bivalves in the Upper Ordovician (see above).

(iii) Pseudocyrtodonta was probably an active infaunal burrower.

(iv) Pseudocyrtodonta shows a very simple hinge like Fasciculodonta. This hinge could be considered close to the ancestral type for the actinodonts, if the group was derived from the cardiolarioids.

\section{Acknowledgements}

I am indebted to Jiři Křǐž, Czech Geological Survey, Prague for valuable suggestions and many constructive comments and to John Cope, National Museum of Wales for helpful improvement of the English and constructive comments that very improve this manuscript. I would like to express many thanks to Vojtěch Turek from National Museum and Martina Korandová from Museum of Dr. Bohuslav Horák in Rokycany, who kindly provided access to their collections. The research was funded by GA ČR (Czech Science Foundation) 205/09/1521, by the Czech Geological Survey in the project 334600 and by IGCP project 591 .

\section{References}

ALLEN, J.A. \& SANDERS, H.L. 1973. Studies on deep-sea Protobranchia (Bivalvia); the families Siliculidae and Lametilidae. Bulletin of the Museum of Comparative Zooology Harvard University 145(6), 263-310.

BABIN, C. 1966. Mollusques Bivalves et Céphalopodes du Paléozoïque armoricain. 470 pp. Imprimerie Commerciale et Administrative, Brest.

BABIN, C. 1982. Mollusques bivalves et rostroconches, 37-49. In Babin, C., Courtessole, R., Melou, M., Pillet, J. \& VizCAÏNO, D. Brachiopodes (articulés) et mollusques (bivalves, rostroconches, monoplacophores, gastropodes) de l'Ordovicien inférieur (Trémadocien-Arenigien) de la Montagne Noire (France méridionale). Mémoire de la Société des Études Scientifiques de l'Aude. 63 pp. Sival, Carcassonne.

BABIN, C. \& GutiÉRREZ-MARCO, J.C. 1985. Un noveau Cycloconchidae (Mollusca Bivalvia) du Llanvirn inférieur (Ordovicien) des Monts de Tolede (Espagne). Geobios 18(5), 609-616. DOI 10.1016/S0016-6995(85)80060-7

BABIN, C. \& GUTIÉRREZ-MARCO, J.C. 1991. Middle Ordovician Bivalves from Spain and their phyletic and palaeogeographic significance. Palaeontology 34(1), 109-147.

BABIN, C. \& LE PENNEC, M. 1982. Ontogénèse et phylogénèse: à propos de quelques caractères dentaires des mollusques bivalves. Malacologia 22(1-2), 709-720.

BARRANDE, J. 1881. Systême silurien du centre de la Bohême. Classe des Mollusques, ordre des Acéphalés 6. 342 pp. Prague \& Paris.

BARROIS, C. 1891. Mémoire sur la faune du Grès armoricain. Annales de la Société géologique du Nord 19, 134-237.

BIELER, R., CARTER, J.G. \& COAN, E.V. 2010. Classification of bivalve families, 113-133. In BOUCHET, P. \& ROCROI, J.P. (eds) Nomenclator of bivalve families. Malacologia 52(2), $1-184$.

Carter, J., Altaba, C.R., Anderson, L.C., Araujo, R., BiaKov, A.S., BOGAN, A.E., CAMPBELl, D.C., CAMPBELl, M., Chen, J., Cope, J.C.W., Delvene, G., DijKstra, H.H., FANG, Z., GARDNER, R.N., GAVRILOVA, V.A., GONCHAROVA, I.A., HARRIES, P.J., HARTMAN, J.H., HAUTMANN, M., HoEH, W.R., HylleberG, J., JiAnG, B., JOHNSTON, P., KIRKENDAle, L., KleEMANN, K., KOPPKA, J., KŘíž, J., MAChado, D., Malchus, N., Márquez-Aliaga, A., Masse, J.-P., MCRoberts, C.A., MidDElfart, P.U., Mitchell, S., NeVesskaja, L.A., Özer, S., POJETA, J. JR., POLUbotKo, I.V., PONS, J.M., POPOV, S., SÁNChez, T., SARTORI, A.F., SCOTT, R.W., SEY, I.I., SigNORELl, J.H., SilANTIEV, V.V., SKelton, P.W., Steuber, T., WATERHOUSE, J.B., WINGARD, G.L. \& YANCEY, T. 2011. A synoptical classification of the Bivalvia (Mollusca). University of Kansas, Paleontological Institute, Paleontological Contributions 4, 1-47.

CARTER, J., CAMPBELl, D.C. \& CAMPBELL, M.R. 2000. Cladistic perspectives on early bivalve evolution, 47-79. In HARPER, E.M., TAYLOR, J.D. \& CRAME, J.A. (eds) The Evolutionary Biology of the Bivalvia. The Geological Society of London, Special Publication 177.

COPE, J.C.W. 1996. Early Ordovician (Arenig) bivalves from the Llangynog Inlier, South Wales. Palaeontology 39(4), 979-1025.

COPE, J.C.W. 1997. The early phylogeny of the class Bivalvia. Palaeontology 40(3), 713-746.

COPE, J.C.W. 2002. Diversification and biogeography of bivalves during the Ordovician Period, 25-52. In CRAME, J.A. \& OWEN, A.W. (eds) Palaeogeography and biodiversity change: the Ordovician and Mesozoic-Cenozoic radiations. Geological Society of London, Special Publications 194.

DECHASEAUX, C. 1952. Classes des Lamellibranches (Lamellibranchiata Blainville, 1816), 220-364. In PIVETEAU, J. (ed.) Traité de Paléontologie 2. Masson et Cie, Paris.

FANG, Z.J. \& COPE, J.C.W. 2004. Early Ordovician bivalves from Dali, West Yunnan, China. Palaeontology 47(5), 1121-1158. DOI 10.1111/j.0031-0239.2004.00403.x

FANG, Z.J. \& COPE, J.C.W. 2008. Affinities and palaeobiogeographical significance of some Ordovician bivalves from East Yunnan, China. Alcheringa 32, 297-312. DOI 10.1080/03115510802096150

FOERSTE, A.F. 1914. The Rogers Gap Fauna of Central Kentucky. Journal of the Cincinnati Society of Natural History 21, 109-156.

GuO, F. 1985. Fossil bivalves of Yunnan. 319 pp. Yunnan Science and Technology publishing house, Kumming, China. [in Chinese]

GuO, F. 1988. New genera of fossil bivalves from Yunnan. Yunnan Geology 7, 112-144. [in Chinese]

JEFFREYS, J.G. 1879. On the Mollusca procured during the 'Lightning' and 'Porcupine' Expeditions, 1868-70. Part II. Proceedings of the Zoological Society of London 1879, 553-588.

JELL, P.A. 1980. Earliest known bivalve on Earth - a new Early Cambrian genus from South Australia. Alcheringa 4, 233-239. DOI 10.1080/03115518008618934

KOROBKOV, I.A. 1954. Handbook of Tertiary molluscs, bivalves. 444 pp. Gostoptex, Leningrad.

KŘíž, J. 1969. Genus Butovicella Kříž, 1965 in the Silurian of Bohemia (Bivalvia). Sborník geologických věd, Palaeontologie 10, 105-139.

KŘíž, J. \& STEINOVÁ, M. 2009. Uppermost Ordovician bivalves from the Prague Basin (Hirnantian, Perunica, Bohemia). Bulle- 
tin of Geosciences 84(3), 409-436.

DOI 10.3140/bull.geosci.1141

LINNÉ, C. 1758. Systema naturae per regna tria naturae, secundum classes, ordines, genera, species, cum characteribus, differentiis, synonymis, locis. Editio decima, reformata. 824 pp. Holmiae.

MAILliEUX, E. 1939. L'Ordovicien de Sart-Bernard. Mémoire du Musée Royal d'Histoire naturelle 86, 1-59.

MCALESTER, A.L. 1968. Type species of Paleozoic nuculoid bivalve genera. Memoir of the Geological Society of America $105,1-143$.

MCALESTER, A.L. 1969. Palaeotaxodonta (Bivalvia), N227-N243. In MOORE, R.C. (ed.) Treatise on Invertebrate Paleontology. Part N, Mollusca 6, Bivalvia. 487 pp. Geological Society of America \& University of Kansas Press, Boulder \& Lawrence.

MEEK, F.B. 1871. A preliminary list of fossils collected by Dr. Hayden in Colorado, New Mexico, and California, with brief descriptions of a few of the new species. Proceedings of the American Philosophical Society 11, 425-431.

MiLlER, S.A. 1874. Monograph of the Lamellibranchiata of the Cincinnati group. Cincinnati Quarterly Journal of Science 1, 211-231.

Miller, S.A. 1889. North American Geology and Palaeontology for the use of Amateurs, Students and Scientists. 664 pp. Western Methodist Book Concern, Cincinnati.

MORRIS, N.J. 1980. A new Lower Ordovician bivalve family, the Thorallidae (?Nuculoida) interpreted as actinodont deposit feeder. Bulletin of the British Museum of Natural History, Geology 34, 265-272.

MORRIS, N.J. \& FORTEY, R.A. 1976. The significance of Tironucula gen. nov. to the study of bivalve evolution. Journal of Palaeontology 50(4), 701-709.

NEUMAYR, M. 1884. Zur Morphologie der Bivalvenschlosses. Sitzungsberichte der Kaiserlichen Akademie der Wissenschaften, Mathematisch-naturwissenschaftliche Klasse 88(2), 385-419.

NEWELL, N.D. 1969. Classification of Bivalvia, N205-N224, N393-N401. In MOORE, R.C. (ed.) Treatise on Invertebrate Paleontology. Part N, Vol. 1. Mollusca 6, Bivalvia. 487 pp. Geological Society of America \& University of Kansas Press, Boulder \& Lawrence.

PELSENEER, P. 1889. Sur la classification phylogénétique de pélécypods. Bulletin Scientifique de la France et de la Belgique, 20 [= sér. 3, 2] (1-4), 27-52.

PFAB, L. 1934. Revision der Taxodonta des böhmischen Silurs. Palaeontographica, Abteilung A 80, 195-253.

PHILLIPS, J. 1848. The Malvern Hills compared with the Palaeozoic districts of Abberley, Woolhope, May Hill, Tortworth, and Usk. Memoirs of the Geological Survey, Great Britain, and of the Museum of Practical Geology in London 2(1), 1-330, with Palaeontological Appendix by J. Phillips and J.W. Salter, 331-386.

PoJETA, J. 1971. Review of Ordovician Pelecypods. United States Geological Survey Professional Papers 685, 1-46.

POJETA, J. 1978. The origin and early taxonomic diversification of Pelecypods. Philosophical Transactions of the Royal Society of London B 284, 225-246. DOI 10.1098/rstb.1978.0065

PojeTA, J. \& GilberT-TOMLinson, J. 1977. Australian Ordovician pelecypod molluscs. Bureau of Mineral Resources, Geology and Geophysics, Bulletin 174, 1-64.

POJETA, J. \& RUNNEGAR, B.N. 1985. The early evolution of diasome Mollusks, 295-336. In TRUMAN, E.R. \& ClARKE, M.R. (eds) The Mollusca. Vol. 10. Evolution. Academic Press, New York.
RATTER, V.A. \& COPE, J.C.W. 1998. New neotaxodont bivalves from Silurian of South Wales and their phylogenetic significance. Palaeontology 41, 975-991.

RoUAULT, R. 1851. Mémoires sur le terrain paléozoïque des environs de Rennes. Bulletin de la Societé Geologique de France 8 , 358-399.

RunNeGAR, B. \& BentLEy, C. 1983. Anatomy, ecology and affinities of the Australian early Cambrian bivalve Pojetaia runnegari. Journal of Paleontology 57(1), 73-92.

SALTER, J.W. 1859. Fossils from the Base of the Trenton Group. Figures and descriptions of Canadian organic remains, Decade 1.47 pp. Geological Survey of Canada, Montreal.

SÁNCHEZ, T.M. 1986. Una fauna de bivalvos en la formacion Santa Gertrudis (Ordovicico) de la provincia de Salta (Argentina). Ameghiniana 23(3-4), 131-139.

SÁNCHEZ, T.M. 1995. Un nuevo genero de Tironuculidae (Bivalvia, Palaeotaxodonta) en el arenigiano del Noroeste Argentino. Geobios 28(6), 683-690. DOI 10.1016/S0016-6995(95)80061-1

SÁNCHEZ, T.M. 1997. Additional mollusca (Bivalvia and Rostroconchia) from the Suri Formation, early Ordovician (Arenig), western Argentina. Journal of Palaeontology 71(6), 1046-1054.

SÁNCHEZ, T.M. 2001. Moluscos bivalvos de la Formación Molles (Arenigiano medio), Sierra de Famatina, Argentina. Ameghiniana 38(2), 185-193.

SÁNCHEZ, T.M. 2005. New Bivalvia and Rostroconchia from the early Ordovician (late Tremadoc-middle Arenig) of northwestern Argentina. Journal of Paleontology 79(3), 532-541. DOI 10.1666/0022-3360(2005)079<0532:NBARFT>2.0.CO;2

SÁNCHEZ, T.M. \& BABIN, C. 1994. Los Géneros Redonia y Catamarcaia (Mollusca, Bivalvia) de la Formación Suri (Ordovícico temprano, oeste de Argentina) y su interés Paleobiogeográfico. Revista Española de Paleontología 9(1), 81-90.

SÁNCHEZ, T.M. \& BABIN, C. 1998. The origin of Actinodont from taxodont dentition or vice-versa: an unnecesssary controversy, 29-51. In TAYLOR, J.D. (ed.) Origin and Evolutionary Radiation of the Mollusca. Oxford University Press, Oxford.

SÁnCHEZ, T.M. \& BEnEDETTO, L.J. 2007. The earliest known estuarine bivalve assemblage, Lower Ordovician of northwestern Argentina. Geobios 40, 523-533.

DOI 10.1016/j.geobios.2006.06.002

SÁnCHEZ, T.M., MARENGO, L. \& Astini, A.R. 2003. Late Ordovician Bivalvia Heteroconchia (Cycloconchidae and Glyptarcidae) from western Argentina. Ameghiniana 40(3), 433-440.

SÁNCHEZ, T.M. \& VACCARI, N.E. 2003. Ucumariidae new family (Bivalvia, Anomalodesmata) and other bivalves from the Early Ordovician (Tremadocian) of northwestern Argentina. Ameghiniana 40(3), 415-424.

ULRICH, E.O. 1893. New and little known Lamellibranchiata from the Lower Silurian rocks of Ohio and adjacent states. Ohio Division of Geological Survey 7, 627-693.

UlRICH, E.O. \& SCOFIELD, W.H. 1894. The Lower Silurian Lamellibranchiata of Minnesota, 475-628. In ULRICH, E.O., SCOFIELD, W.H., Clarke, J.M. \& WinCHELL, N.H. (eds) Final Report of the Geological and Natural History Survey of Minnesota, vol. 3, part 2, The Geology of Minnesota. Paleontology. Published and distributed under separate cover prior to publication of entire vol. 3 in 1897. Systematics of Bivalvia by Ulrich alone.

WALLER, T.R. 1990. The evolution of ligament systems in the Bivalvia, 49-71. In MORTON, B. (ed.) The Bivalvia. Proceedings of a memorial symposium in honour of Sir Charles Maurice Yonge (1899-1986), Edinburgh 1986, 1-355. Hong Kong University Press, Hong Kong. 